\title{
Modelling and Identification of Underwater Glider for Heading Angle Correction
}

\author{
Tri Wahyu O. Putri, Ulinnuha Latifa, Bambang R. Trilaksono, and Egi M. I. Hidayat
}

\begin{abstract}
Autonomous Underwater Glider (AUG) as a high durability Autonomous Underwater Vehicle (AUV) is typically capable of extremely long missions and may travel thousands of kilometers in the ocean. An ability to track the desired paths is indispensable ability of underwater glider due to its long range paths. Heading control system has the main role in path tracking. This paper presents mathematical modelling of a class of underwater glider in lateral plane in order to design the heading control system. Model identification using N4SID method was used to tune the parameters based on the experiment data. The heading motion model of a class underwater glider was evaluated in simulation with varying inputs. The results showed that the tuned model's responses satisfy the real behavior of underwater glider.
\end{abstract}

Index Terms-Autonomous underwater glider, modelling, identification, heading motion.

\section{INTRODUCTION}

The ocean covers $71 \%$ of Earth's surface. It is important to observe both physical and biological dynamics of the ocean. The main challenge is collecting data "in a vast, inhospitable, and unforgiving" ocean environment [1]. In recent years, oceanographers have used Autonomous Underwater Vehicle (AUV) as an important tool in gathering oceanographic data, replacing the traditional process [1]. Conventional AUVs use propellers as their main driver and battery as their main power source, which make it cannot be operated for long-term deployment.

Underwater gliders are kind of AUV that propel themselves by modifying the buoyancy and center of mass. It provides the high endurance and long-term operation in ocean research missions. An ability to track the desired paths is indispensable ability of underwater glider. Heading control system has the main role in path tracking. Hence a rudder is an appropriate choice for heading control [2], besides the tunnel thruster that also can be used as heading actuator. Before the rudder and heading control system can be designed and analyzed, the dynamics that describe the motion of a glider in lateral plane must be determined [2]. Derivation of mathematical equations is a prevalent method in modelling. The same modelling method has been used by [3] to their AUG named NPS AUV II. The detail of an AUV's mathematical models has also been

Manuscript received March 10, 2017; revised April 24, 2017. This work was supported in part by School of Electrical Engineering and Informatics of Institut Teknologi Bandung and Indonesian Agency for Meterorology, Climatology, and Geophyscs.

T. W. O. Putri, U. Latifa, B. R. Trilaksono are with Electrical Enginnering in Institut Teknologi Bandung, Indonesia (e-mail: tri.wahyu.op@gmail.com, briyanto@1skk.ee.itb.ac.id, egi.hidayat@1skk.ee.itb.ac.id). explained by [4] in their book.

This paper presents underwater glider models of a class of underwater glider in the lateral plane in order to design the heading control system, based on the underwater glider that we built, named GaneshBlue Glider. Modelling in the lateral plane was also presented and succesfully applied by [5] to their HUGIN 4500 AUV. It is an undeniable truth that every system, in this case AUV, has different model one to another because of different dimensions, shapes, physical characteristics, and actuators. The models which were presented in many literatures were not suitable if applied in GaneshBlue due to the differences in physical parameters and actuators. Then, it needs to build the right models for it.

The paper is organized as follows. Section 2 gives a system overview of the GaneshBlue Glider and its control systems. Section 3 focuses in modelling. Linearization of dynamic models and parameter determination using analytical methods are described in this section as well. Section 4 describes the identification method. Section 5 shows the simulation result based on the tuned model with doublet input. Section 6 provides concluding remarks and directions for the future works.

\section{SYSTEM OVERVIEW}

\section{A. GaneshBlue Glider}

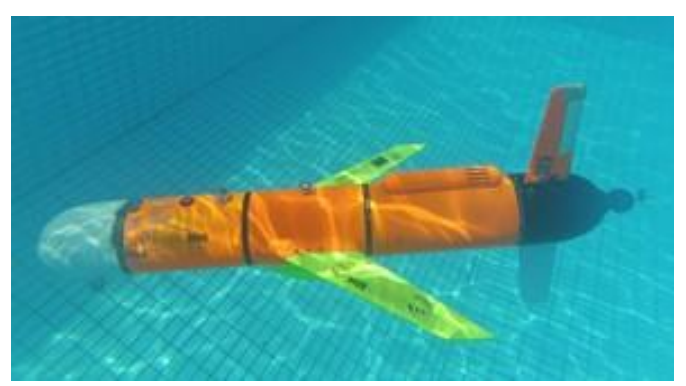

Fig. 1. GaneshBlue Glider.

GaneshBlue Glider is buoyancy-driven underwater glider which was developed by Institut Teknologi Bandung, Indonesia. This glider is designed for shallow water exploration with maximum depth of 200 meters. As shown in Figure 1, this underwater glider has 2 meter's length, $92.8 \mathrm{~kg}$ nominal dry mass, and propels itself by modifying the buoyancy and center of mass. The buoyancy is controlled by a bladder bag which can modify its buoyancy due to volume displacement. The pitch angle is controlled by two ballast tanks inside the hull. The motion in the lateral plane of GaneshBlue Glider is actuated using a rudder and two horizontal tunnel thrusters. This paper is focused in 
underwater glider motion in the lateral plane with a rudder as the heading actuator.

\section{B. Heading Control}

The most intuitive means of changing heading is by deflecting a rudder to induce a yawing moment [2] or by using two horizontal thrusters (bow thruster and stern thruster). GaneshBlue Glider has a rudder in the upper vertical wing and two horizontal tunnel thrusters which are located in rear and front. The maximum rudder deflection angle is \pm 30 degrees. Controller design of heading control is important part to correct the heading angle in order to keep the glider tracks the desired path.

\section{Model of AUG IN LAteral Plane}

Model of AUG in lateral plane can be developed from its kinematic and dynamic equations. Then, the value of hydrodynamic parameters are determined by hydrodynamic parametrization using analytical methods. After that, the model of a class off AUG in lateral plane have to be validated by comparing its model response with experiment's response.

\section{A. Kinematic Equation}

The forces and moments acting on the underwater glider need to be referenced to the same frame [3]. This paper uses standard notation from [4] to describe the 6 DOF (Degree of Freedom) equations of motion. AUG motion in lateral plane neglects any other motions and rotations in longitudinal plane. Then the kinematic equations in lateral plane are as follow (see (1) and (2)), with $x$ is position in x-direction, $\dot{x}$ is linear velocity along $\mathrm{x}$-axis in world coordinate, $y$ is position in y-direction, $\dot{y}$ is linear velocity along $y$-axis in world coordinate, $\psi$ is yaw angle, $u$ is surge velocity along $\mathrm{x}$-axis in body coordinate, and $v$ sway velocity along $\mathrm{x}$-axis in body coordinate. The $x$ and $y$ axes of AUG which is seen from above is described as in Fig. 2.

$$
\begin{aligned}
& \dot{x}=u \cos \psi-v \sin \psi \\
& \dot{y}=u \sin \psi+v \cos \psi
\end{aligned}
$$

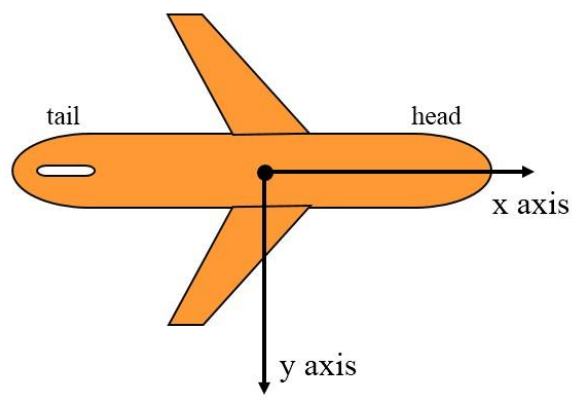

Fig. 2. $x$ and $y$ axes of AUG.

\section{B. Dynamic Equation}

Dynamic equations in the lateral plane are sway force equation (see (3)) and yaw moment equation (see (4)). It is well-known [3]-[5] that speed can be decoupled from sway and yaw dynamics. The dynamics equations in the lateral plane [4]-[6] are shown in equation below.

$$
\begin{aligned}
& m\left[\dot{v}+u r+x_{G}(p q+\dot{r})-y_{G}\left(p^{2}+r^{2}\right)+x_{G}(q r+\dot{p})\right] \\
& =Y_{H}+Y_{W}+Y_{C} \\
I_{z} & \dot{r}+\left(I_{y}-I_{x}\right) p q-I_{x y}\left(p^{2}-q^{2}\right)+m\left[x_{G}(\dot{v}+u r)-y_{G}(\dot{u}-v r)\right] \\
= & N_{H}+N_{W}+N_{C}
\end{aligned}
$$

where $m$ is AUG mass, $x_{G}$ is x position of center of gravity, $y_{G}$ is y position of center of gravity, $r$ is yaw angular velocity, $p$ is roll angular velocity, $q$ is pitch angular velocity, $I_{z}$ is inertia of vehicle in $\mathrm{z}$ axis, $I_{x}$ is inertia of vehicle in $\mathrm{z}$ axis, $I_{y}$ is inertia of vehicle in y axis, $I_{x y}$ is x-y cross inertia, and the right side of those equations are external forces.

Hydrodynamic force and moment:

$$
\begin{aligned}
& Y_{H}=D_{4}\left(Y_{\dot{r}} \dot{r}+Y_{q r} q r\right)+D_{3}\left(Y_{\dot{v}} \dot{v}+Y_{r} u r+Y_{v q} v q+Y_{w r} w r\right) \\
& +D_{2}\left(Y_{v} u v+Y_{v w} v w\right) \\
& N_{H}=D_{5}\left(N_{\dot{r}} \dot{r}+N_{q r} q r\right)+D_{4}\left(N_{\dot{v}} \dot{v}+N_{r} u r+N_{v q} v q+N_{w r} w r\right) \\
& +D_{3}\left(N_{v} u v+N_{v w} v w\right) \\
& Y_{\dot{r}}, Y_{q r}, Y_{\dot{v}}, Y_{r}, Y_{v q}, Y_{w r}, Y_{v}, Y_{v w}, N_{\dot{r}}, N_{q r}, N_{\dot{v}}, N_{r}, N_{v q}, N_{w r}, N_{v}, N_{v w}
\end{aligned}
$$
are hydrodynamic coefficients and $D_{n}=0.5 \rho L^{n}$ where $\rho$ is water density, $L=2 \mathrm{~m}$ is the vehicle length.

Hydrostatic forces and moments for motion in the lateral plane are described in Equation 7 and 8, where $g$ is gravity acceleration, $V_{v}$ is AUG's volume, $\theta$ is pitch angle, $\phi$ is roll angle, $x_{B}$ is $\mathrm{x}$ position of vehicle's center of buoyancy, and $y_{B}$ is y position of vehicle's center of buoyancy.

$$
\begin{gathered}
Y_{W}=\left(m g-\rho g V_{v}\right) \cos \theta \sin \phi \\
N_{W}=\left(x_{G} m g-x_{B} \rho g V_{v}\right) \cos \theta \sin \phi+\left(y_{G} m g-y_{B} \rho g V_{v}\right) \sin \theta
\end{gathered}
$$

Force and moments due to rudder deflection and tunnel thrusters are described in Equations 9 and 10.

$$
\begin{gathered}
Y_{C}=D_{2} u^{2}\left(Y_{\delta r} \delta r\right) \\
N_{C}=D_{3} u^{2}\left(N_{\delta r} \delta r\right)+L_{t 1} F_{t 1}+L_{t 2} F_{t 2}
\end{gathered}
$$

where, $\delta_{r}$ is rudder angle deflection, $L_{t l}$ is distance of bow tunnel thruster to center of gravity of underwater glider, $F_{t l}$ is bow tunnel thruster's force, $L_{t 2}$ is distance of stern tunnel thruster to center of gravity of underwater glider, $F_{t 2}$ is stern tunnel thruster's force.

Assumed that in the lateral motion, roll angle is equal to zero. Then the nonlinear dynamic equations of heading motion are derived from Equation 4 with assumption that the sway force is less significant in heading model.

$$
\begin{aligned}
& \left(I_{z}-D_{5} N_{\dot{r}}\right) \dot{r}-m y_{G} \dot{u}=\left(y_{G} m g-y_{B} \rho g V_{v}\right) \sin \theta \\
& +\left(D_{4} N_{r} u-m x_{G} u\right) r+D_{4} N_{w r} w r-I_{x y} q^{2} \\
& +D_{3} u^{2}\left(N_{\delta r} \delta r\right)+L_{t 1} F_{t 1}+L_{t 2} F_{t 2}
\end{aligned}
$$

\section{Model Linearization}

Model linearization is needed because Equation 11 is a nonlinear dynamic equation, while this paper's objective is to 
obtain the linear heading model of a class of underwater glider. Linearization uses Taylor's Series Method is described as follows:

$$
\begin{gathered}
\dot{r}=\left\lfloor\frac{\partial f}{\partial r}\right\rfloor_{\left(r_{0}, U_{0}\right)} r+\left\lfloor\frac{\partial f}{\partial U}\right\rfloor_{\left(r_{0}, U_{0}\right)} U \\
U=\left(\delta_{r}, F_{t 1}, F_{t 2}\right) \\
\dot{r}=\left.\frac{\partial f}{\partial r}\right|_{\left(r_{0}=0\right)} r+\left[\begin{array}{lll}
\frac{\partial f}{\partial \delta_{r}} & \frac{\partial f}{F_{t 1}} & \frac{\partial f}{F_{t 2}}
\end{array}\right]_{\left(r_{0}, U_{0}=0\right)}\left\lfloor\begin{array}{l}
\delta_{r} \\
F_{t 1} \\
F_{t 2}
\end{array}\right\rfloor
\end{gathered}
$$

Thus the linear dynamics heading model in the lateral plane are described in Equation 14 with assumption $\sin \theta=\theta, \cos \theta=1$, $\delta_{r 0}=F_{t 1_{0}}=F_{t 2_{0}}=r_{0}=y_{G}=y_{B}=x_{G}=x_{B}=0$.

$\left[\begin{array}{c}\dot{\psi} \\ \dot{r}\end{array}\right]=\left[\begin{array}{cc}0 & 1 \\ 0 & \frac{D_{4} N_{r} u}{I_{z}-D_{5} N_{\dot{r}}}\end{array}\right]\left[\begin{array}{l}\psi \\ r\end{array}\right]+\left[\begin{array}{ccc}0 & 0 & 0 \\ \frac{D_{3} N_{\delta r} u^{2}}{I_{z}-D_{5} N_{\dot{r}}} & \frac{L_{t 1}}{I_{z}-D_{5} N_{\dot{r}}} & \frac{L_{t 2}}{I_{z}-D_{5} N_{\dot{r}}}\end{array}\right]\left[\begin{array}{c}\delta_{r} \\ F_{t 1} \\ F_{t 2}\end{array}\right]$

Physical and system parameters of linear heading model in the lateral plane as described in Equation 14 are shown in Table I.

TABLE I: PHYSICAL AND SYSTEM PARAMETERS OF LINEAR HEADING

\begin{tabular}{ll}
\multicolumn{2}{c}{ MODEL } \\
\hline \hline Parameters & Value \\
\hline$m$ & $92.8 \mathrm{Kg}$ \\
$u$ & $0.3 \mathrm{~m} / \mathrm{s}$ \\
$I_{z}$ & $26.574 \mathrm{Kg} \cdot \mathrm{m}^{2}$ \\
$L_{t 1}$ & $0.917 \mathrm{~m}$ \\
$L_{t 2}$ & $0.748 \mathrm{~m}$ \\
\hline \hline
\end{tabular}

\section{Hydrodynamic Parametrization}

Hydrodynamics parametrization using analytical methods based on [7] and [8] gives values for hydrodynamic parameters for heading motion in the lateral plane (see Table II).

TABLE II: HydRODYNAMIC PARAMETERS FOR HEADING MOTION MODEL

\begin{tabular}{ll}
\hline \hline Parameters & Value \\
\hline$N_{\delta r}$ & -0.0158 \\
$N_{r}$ & -0.0170 \\
$N_{\dot{r}}$ & $7.275 \times 10^{-4}$ \\
$D_{3}$ & 4000 \\
$D_{4}$ & 8000 \\
$D_{5}$ & 16000 \\
\hline \hline
\end{tabular}

\section{E. Model Validation}

Using given model and parameters, the response of heading motion model in the lateral plane can be simulated using Matlab as seen in Figure 3. The simulation and validation only used rudder deflection angle as the input. So, the equation (14) can be simplified as single input and single output state space. The input is rudder deflection angle and the output is yaw (heading) angle. Henceforth, this paper focused in heading model with rudder deflection angle as its input.

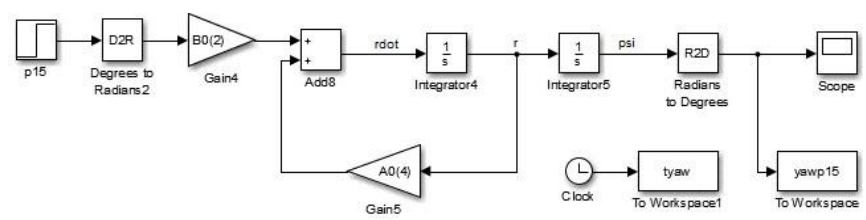

Fig. 3. Model simulation using Simulink Matlab.

The model's response was compared with the experiment data. Experiment data were gotten from pool testing using the real GaneshBlue Glider with various rudder deflection angle. Figure 4 shows the comparison of heading model as validation of model. It can be concluded from the plots that there were big differences between model and real data. Thus, the heading model needed to be tuned by identification method.

\section{IDENTIFICATION}

The identification method that was used in this paper is N4SID. N4SID stands for Numerical algorithms for Subspace State Space System Identification. This method is well-known as powerful method for high order multivariable systems. With N4SID algorithms, most of a priori parametrization can be avoided. Only the order of the system is needed and it can be determined through inspection of the dominant singular values of a matrix that is calculated during identification [9].
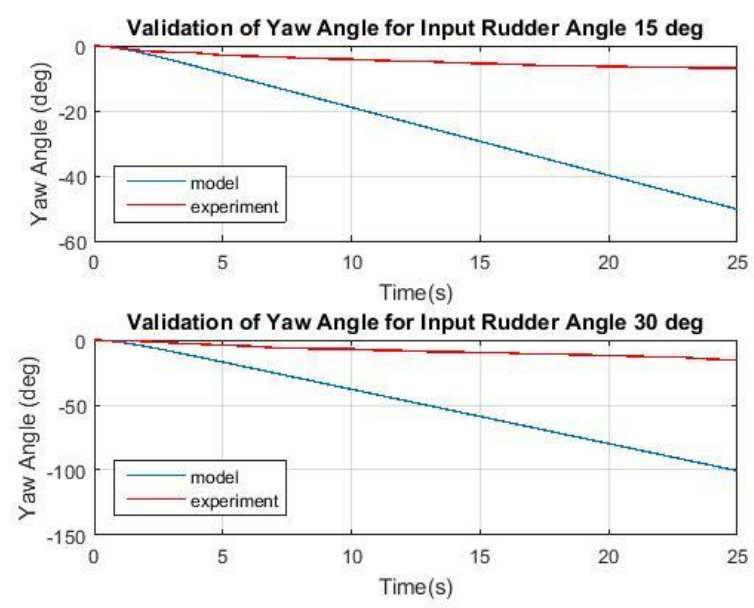

Fig. 4. Validation of model for input rudder angle 15 and 30 degree.

N4SID solves subspace matrix equations by estimating past and future of Kalman filter states. The detail of N4SID algorithm can be seen in [9]-[11].

With the toolbox of Matlab ident, it is possible to identify a model with different types of structures using N4SID method. The $n 4$ sid syntax in Matlab estimates models in state space form. The identification was done using input and output experiment data from pool testing. The response of raw data needed to be linearized in order to avoid N4SID estimating the model as first order system whose steady state value. The response of heading model is supposed to be ramp. Figure 5 shows the result of linear regression of yaw (heading) angle response with various rudder angle.

The input and output data that were used to estimate is showed in Fig. 6, which the data were input-output data with rudder deflection angle is -15 degree (0.26 radian). The identification using N4SID method gave a fit value of $100 \%$ 
(see Fig. 7). The cross validation of model estimation with another input-output data shows a fit value of $94.61 \%$ (see Fig. 8).
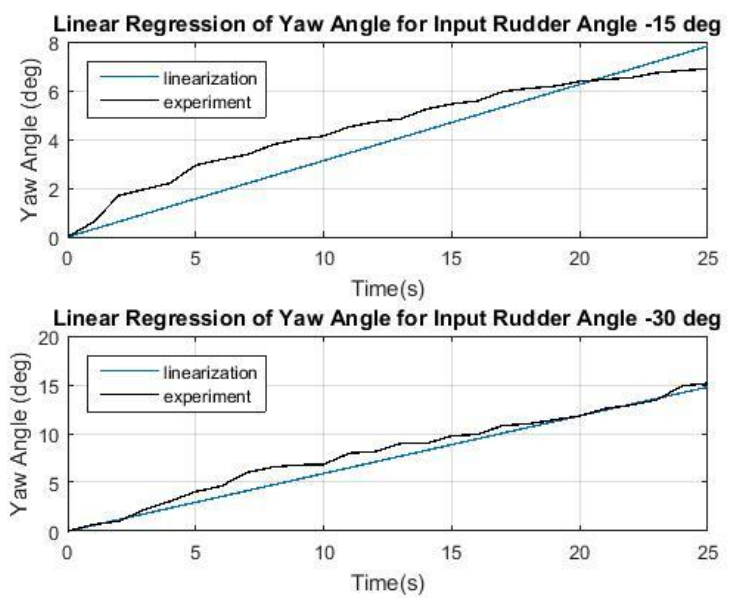

Fig. 5. Linear regression of output data with various rudder angles.

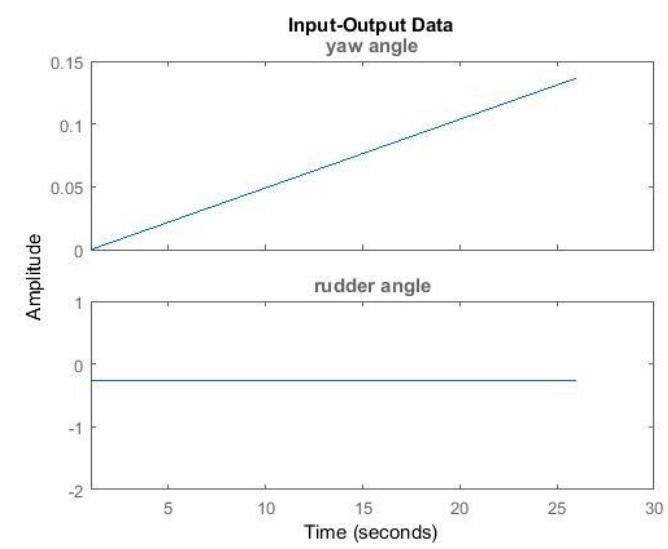

Fig. 6. Input and output data for identification.

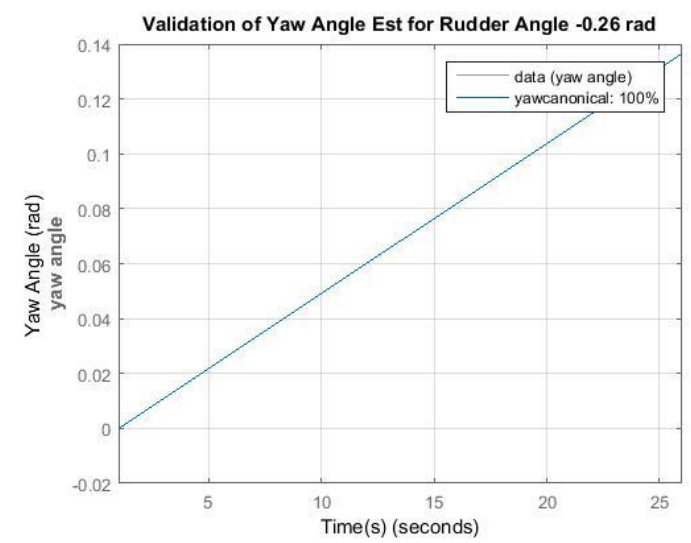

Fig. 7. Validation of heading angle estimation and experiment data for input rudder angle -0.26 radian.

New parameters were added to compensate the difference between model estimation and original model. The tuned model with added parameters is described in (15). The values of added parameters are $K_{y 1}=1.866 \times 10^{-8} ; K_{y 2}=1.75704 ; K_{y 3}$ $=-0.02086 ; K_{y 4}=-2.8594 \times 10^{-8}$.

$$
\left[\begin{array}{c}
\dot{\psi} \\
\dot{r}
\end{array}\right]=\left[\begin{array}{cc}
0 & 1 \\
K_{y 1} & \frac{D_{4} N_{r} u}{I_{z}-D_{5} N_{\dot{r}}} K_{y 2}
\end{array}\right]\left[\begin{array}{c}
\psi \\
r
\end{array}\right]+\left\lfloor\begin{array}{c}
K_{y 3} \\
\frac{D_{3} N_{\delta r} u^{2}}{I_{z}-D_{5} N_{\dot{r}}} K_{y 4}
\end{array}\right\rfloor \delta_{r}
$$

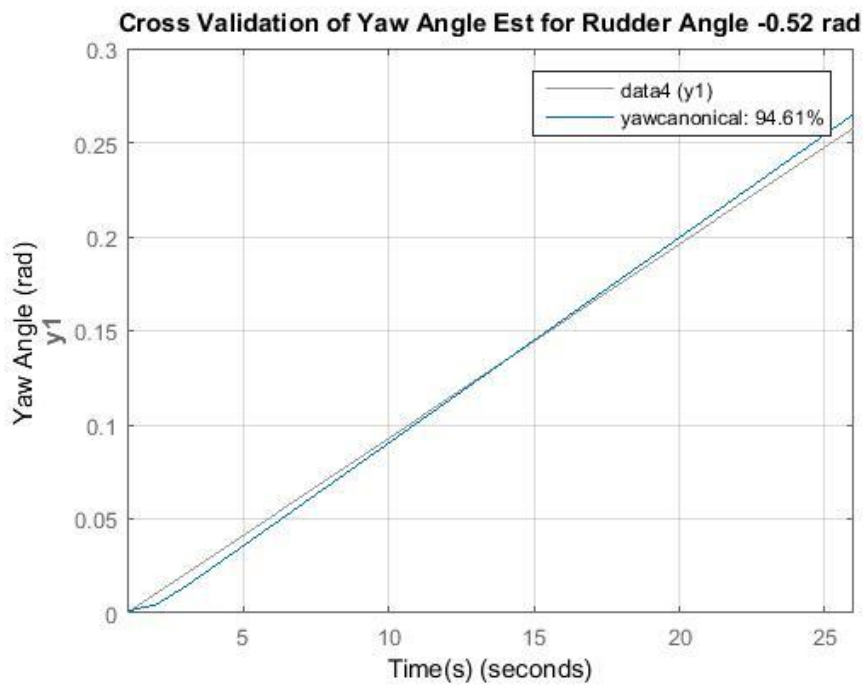

Fig. 8. Cross validation of heading angle estimation and experiment data for input rudder angle -0.56 radian.

\section{Simulation}

The simulation of a class of underwater glider's heading model was built in Matlab to observe the system's response with certain input. Basically, the simulation blocks were almost similar to the simulation blocks in Figure 3 except the addition of parameter compensations. The output of heading angle with doublet signal as an input had been simulated. The result is shown in Fig. 9. The simulation using tuned model were compared with experiment data are shown in Fig. 10 and 11. The simulation shows that the tuned model's responses satisfy the real behavior of underwater glider.
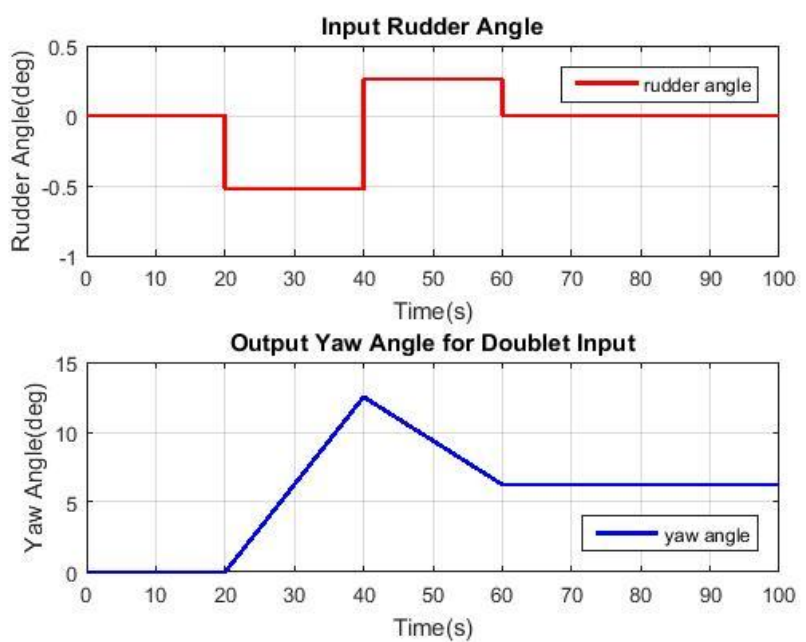

Fig. 9. Input and output of heading (Yaw) angle using D oublet input.

The heading angle correction using the obtained heading model is proposed to keep the AUG tracks the desired trajectory. It is the complex system which consists of guidance system, navigation system, and heading control system. The model is used to design the proper control system and to simulate the overall systems before the deployment in the ocean. 


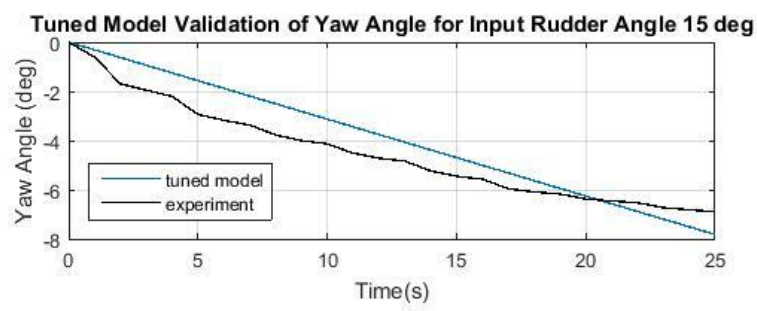

Tuned Model Validation of Yaw Angle for Input Rudder Angle 30 deg

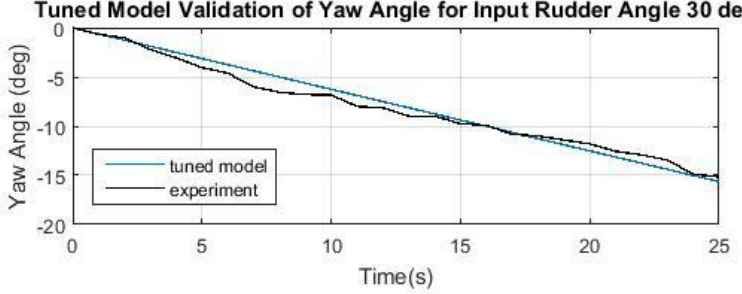

Fig. 10. Tuned model validation of heading model simulation for input rudder angle 15 and 30 degrees.
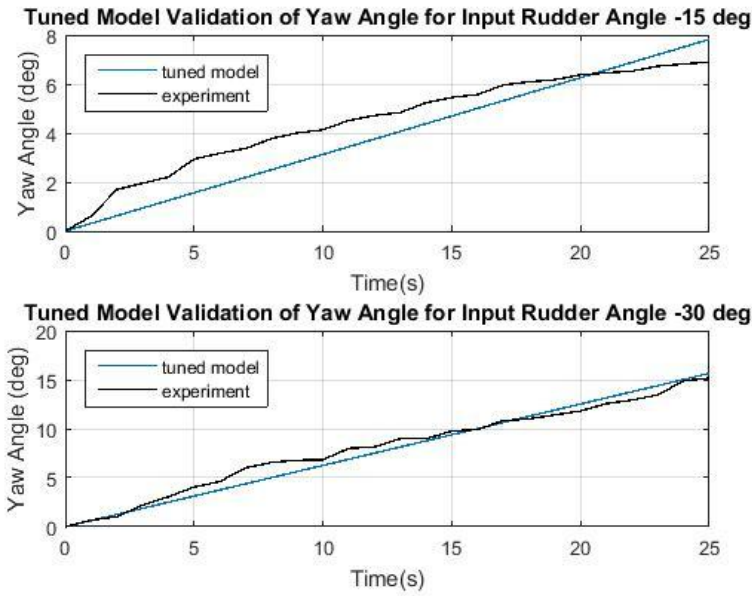

Fig. 11. Tuned model validation of heading model simulation for input rudder angle -15 and -30 degrees.

\section{CONCLUSION}

The models of a class of underwater glider in the lateral plane are presented. Mathematical modelling of heading motion has been developed. The hydrodynamic parameters using analytical methods have been calculated. The model simulation showed there was a steady state error compared with the real data. Identification method using N4SID was used to estimate the suitable model to get the value of added parameters. The model with added parameter had a fit value of $100 \%$. Cross validation of the tuned model also gave satisfying result with a fit value of $94.61 \%$. The simulation showed that the tuned model's responses satisfy the real behavior of underwater glider.

The models of a class of underwater glider which have been developed can be used to design the control system. Guidance and navigation visualization systems can be developed based on the models. Simulation that is built by the models of the underwater glider will eliminate the need for a dedicated and costly support ship during the real experiments and deployment. Moreover, simulation can provide the efficient way to develop the control design algorithm and observe underwater glider's performances.

\section{REFERENCES}

[1] M. Nina. "Efficient motion planning and control for underwater gliders," Diss. Virginia Tech, 2009.

[2] B. Asher et al., "Analysis of an autonomous underwater glider," in Proc. the Australasian Conference on Robotics and Automation, 2008, pp. $1-10$.

[3] F. Geridonmez, "Simulation of motion of an underwater vehicle," Diss. master thesis, Aerospace Engineering Department, Middle East, 2007.

[4] T. I. Fossen, "Marine control systems: Guidance, navigation and control of ships, rigs and underwater vehicles," Marine Cybernetics, Trondheim, Norway, 2002.

[5] F. Magne et al., "Estimation of AUV dynamics for sensor fusion," in Proc. the 2007 10th International Conference on Information Fusion, IEEE, 2007.

[6] I. F. Thor, "Guidance and control of ocean vehicles," John Wiley \& Sons Inc, 1994.

[7] F. A. Ivery, "Simulation, modelling, and control of a near-surface underwater vehicle," PhD diss., University of British Columbia, 2000.

[8] P. Mora et al., "Multiobjective optimization of a submarine hull design," Ship Science and Technology, 2013, pp. 27-42.

[9] P. V. Overschee and D. B. Moor, "N4SID: Subspace algorithms for the identification of combined deterministic - Stochastic systems," Automatica, 1994, vol. 30, no. 1, pp. 75-93.

[10] S. D. M. Borjas and C. Garcia, "Subspace identification for industrial processes," 2011, vol. 12, no. 3, pp. 183-194.

[11] P. Trnka, "Subspace identification methods," Technical Report. Czech Technical University in Prague, Prague, Czech Republic, 2005.

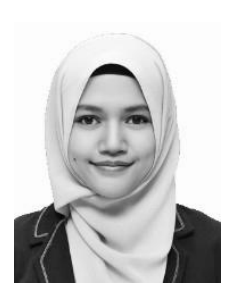

Tri W. O. Putri was born in 1992. She is currently working toward her master degree in control and intelligent system, School of Electrical Engineering and Informatics of Institut Teknologi Bandung, Indonesia. She received her bachelor in control engineering from the Department of Electrical Engineering, Universitas Brawijaya, Malang, Indonesia, in 2014. From 2011 to 2013, she was an assistant in the Control Laboratory of Electrical Engineering Department, Universitas Brawijaya, Malang, Indonesia.

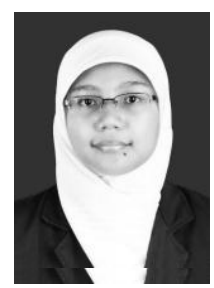

Ulinnuha Latifa was born in 1991. She is currently working toward her master degree in control and intelligent system, School of Electrical Engineering and Informatics of Institut Teknologi Bandung, Indonesia. She received her bachelor in electrical engineering, Universitas Diponegoro, Semarang, Indonesia, in 2013. From 2011 to 2013, she was an assistant in the Control Laboratory of Electrical Engineering Department, Universitas Diponegoro,

Semarang, Indonesia.

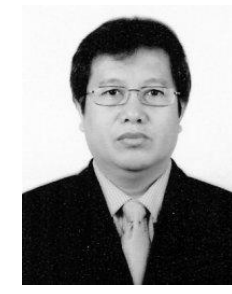

Bambang R. Trilaksono was graduated from Electrical Engineering Dept, Institut Teknologi Bandung (ITB), Indonesia, in 1986. He obtained his master and doctoral degrees both from Electrical Engineering Department, Waseda University, Japan, in 1991 and 1994. He is a lecturer at School of Electrical Engineering and Informatics, ITB. His research interests include robust and intelligent control, multi-agent systems and robotics. He is an advisory committee member of Asian Control Association

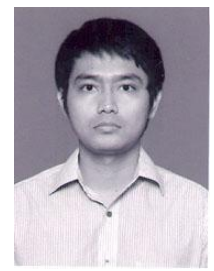

Egi M. I. Hidayat is currently working as lecturer at Institut Teknologi Bandung, Bandung, Indonesia. He received the Ph.D degree in electrical engineering with specialization in Automatic Control from Uppsala University, Sweden, in 2014. He received his M.Sc in control and information system from University of Duisburg-Essen, Germany, in 2007. He was researcher at Uppsala University in research on identification of bilinear wiener systems with Unknown Time Delay. His research interests are focused on system identification and signal processing. 